Proceedings

\title{
SARS-COV-2 serological profile in healthcare professionals and a preliminary evaluation of IgG and IgA in saliva samples: a study from a southern Italy hospital.
}

\author{
Armando De Carlo, Sergio Lo Caputo, Carmela Paolillo, Anna Maria Rosa, Umberto D'Orsi, Pierluigi Reveglia, \\ Teresa Antonia Santantonio and Gaetano Corso * \\ * Correspondence: gaetano.corso@unifg.it
}

\begin{abstract}
Severe acute respiratory syndrome coronavirus 2 (SARS-CoV-2) is the first pandemic caused by a coronavirus. Assessing the prevalence of anti-SARS-CoV-2 in healthcare workers groups offers a unique opportunity to study the correlation between seroconversion and immunization because of their occupational exposure and higher risk of contagious. Methods: The study enrolled 3242 asymptomatic employees of "Policlinico Riuniti," Foggia. After the first screening, we collected sequential serum samples up to 23 weeks from the same subjects. In order to perform a longitudinal follow-up study and get information about the titration of IgG level, we analyzed data from subjects (33) with at least two consecutive serological IgG positive tests. Moreover, we tested some salivary samples analyzing IgG by CLIA method, and IgA by ELISA method. Results: 62 subjects were positive $(1.9 \%, 1.4-2.3 \%, 95 \% \mathrm{CI})$ for at least one antibody anti-SARS-CoV-2. The seroprevalence was lower in the high-risk group $1.4 \%(6 / 428,0.5-2.6 \%, 95 \% \mathrm{CI})$ vs. intermediate-risk group $2.0 \%$ (55/2736, 1.5-2.5\%, 95\% CI). Overall, within eight weeks, we detected a mean reduction of $-17 \%$ in the IgG level. Our data suggest a reduction of about $9.27 \mathrm{AU} / \mathrm{mL}$ every week (R2=0.35, $\mathrm{p}=0.0003$ ). This study revealed a low prevalence of SARS-CoV-2 antibodies among our hospital healthcare staff $(1.9 \%)$. The preliminary analysis of saliva, belonging to positive subjects by molecular testing for SARS CoV2, showed that out of 4 saliva samples we observed an Ig ratio (Ig saliva/Ig Serum) corresponding to $0.06,0.02,0.01,0.03$ for $\operatorname{IgG}$, while it was $2.43,2.42,0.48$, and 1.28 for $\operatorname{IgA}$, respectively. Conclusions: The IgG level reduction suggests that the serological response fades fast in asymptomatic infections. Our preliminary data also suggest that the IgA anti-SARS-CoV2 is present also in saliva of positive subjects and its level is higher than IgG.
\end{abstract}

Keywords: COVID-19; IgA; IgG; Saliva

Publisher's Note: MDPI stays neu-

tral with regard to jurisdictional

claims in published maps and insti-

tutional affiliations.

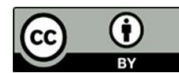

Copyright: (c) 2020 by the authors. Submitted for possible open access publication under the terms and conditions of the Creative Commons Attribution (CC BY) license (http://creativecommons.org/licenses /by/4.0/). 